\title{
Second-order Phase Transition Behavior behind Polymer Glass Transition
}

\author{
Mitsuru Ishikawa,* Taihei Takahashi, Yu-ichiro Hayashi, Maya Akashi, Takayuki Uwada \\ Department of Chemistry, Faculty of Science, Josai University, 1-1 Keyaki-dai, Sakado, Saitama \\ 350-0295, Japan
}

\begin{abstract}
Glass transition has similarity to the second-order phase transition in temperature dependent changes in entropy, non-Arrhenius viscosity, and heat capacity of glass forming materials. However, it has primarily been considered to be not phase transition. Recent singlemolecule spectroscopy developments prompted re-investigating glass transition at the nanometer scale probing resolution, showing that glass transition includes phenomena similar to the secondorder phase transition. They are characterized by microscopic collective polymer motion and discontinuous changes in temperature dependent relaxation times, the latter of which resembles the critical slowing down of second-order phase transitions, within a temperature window above the polymer calorimetric glass transition temperature. Simultaneous collective motion and critical slowing down occurrences disclose that the second-order phase transition hides behind polymer glass transition.
\end{abstract}

\section{INTRODUCTION}

Glass transition differs from phase transition mainly in cooling-rate dependent vitrification temperatures denoted by calorimetric glass transition temperature $T_{\mathrm{g}}$ and its analogue $T_{\mathrm{g}}^{\prime}$. However, we found substantial evidence that second-order phase transitions hide behind polymer glass transition. No one probably feel absurd to hear such statement considering the following glass transition fundamentals showing glass transition similarity to second-order phase transitions.

Figure 1A includes liquid to crystal transition as a first-order phase transition below melting point $T_{\mathrm{m}}$, vitrification at $T_{\mathrm{g}}$, and cooling-rate dependent $T_{\mathrm{g}}$ and $T_{\mathrm{g}}{ }_{\mathrm{g}}$; the difference between them can be $3-5 \mathrm{~K}$ on changing cooling rate by an order of magnitude. ${ }^{1}$ The cooling-rate dependence repels glass transition being a phase transition. However, this figure suggests that glass transition resembles second-order phase transitions. This statement defines second-order phase transition behavior in the present work. Glass transition is not first-order phase transition in the sense that temperature dependent $S$ continues from liquid to glass state without discontinuous transition at $T_{\mathrm{g}}$, although the slopes differ either side of $T_{\mathrm{g}}$. Similarly, extrapolated $S$ profile down to lower temperatures crosses but does not overtake crystal profile at the expected Kauzmann temperature $T_{\mathrm{k}}$ again without discontinuous transition. We learn more second-order phase transition behavior in Figure 1B, showing Arrhenius $\eta$ traces for many glass forming materials called Angell plot. ${ }^{2}$ Those for organic molecules, non-linear or non-Arrhenius ones, fit the Vogel-Fulcher-Tammann (VFT) equation (eq 1) between $T_{\mathrm{g}}$ and $T_{\mathrm{g}}+50 \mathrm{~K},{ }^{3}$ suggesting that glass forming material viscosity $\eta$ diverges at $T_{\mathrm{o}}$ like order parameter divergence at the critical temperature $T_{\mathrm{c}}$ in second-order phase transitions,

$$
\log \left(\eta / \eta_{\infty}\right)=B /\left(T-T_{o}\right),
$$

where $\eta_{\infty}$, the limiting viscosity at infinite temperature; $T_{\mathrm{o}}$, the Vogel temperature, several tens $K$ below $T_{\mathrm{g}} ;{ }^{3}$ and $B$ and $T_{\mathrm{o}}$, empirical parameters fitted from experimental observations.

In addition to second-order phase transition behavior from Figures $1 \mathrm{~A}$ and $1 \mathrm{~B}$, we know one more second-order phase transition behavior characterized by discontinuous change in temperature-dependent heat capacity $C_{\mathrm{p}}=T(\partial S / \partial T)_{\mathrm{p}}$ evaluated by differential scanning calorimetry (DSC) to identify $T_{\mathrm{g}}$, where a single staircase step in $\Delta C_{\mathrm{p}}$ occurs due to the 
discontinuous change in $(\partial S / \partial T)_{\mathrm{p}}$ between liquid and glass state. The transition interval $\Delta T$ associated with $\Delta C_{\mathrm{p}}$ step looks rather small, typically $10 \mathrm{~K}$ for many glass forming materials. ${ }^{3} \mathrm{In}$ general, $\Delta C_{\mathrm{p}}$ step characterizes second-order phase transitions. ${ }^{4}$
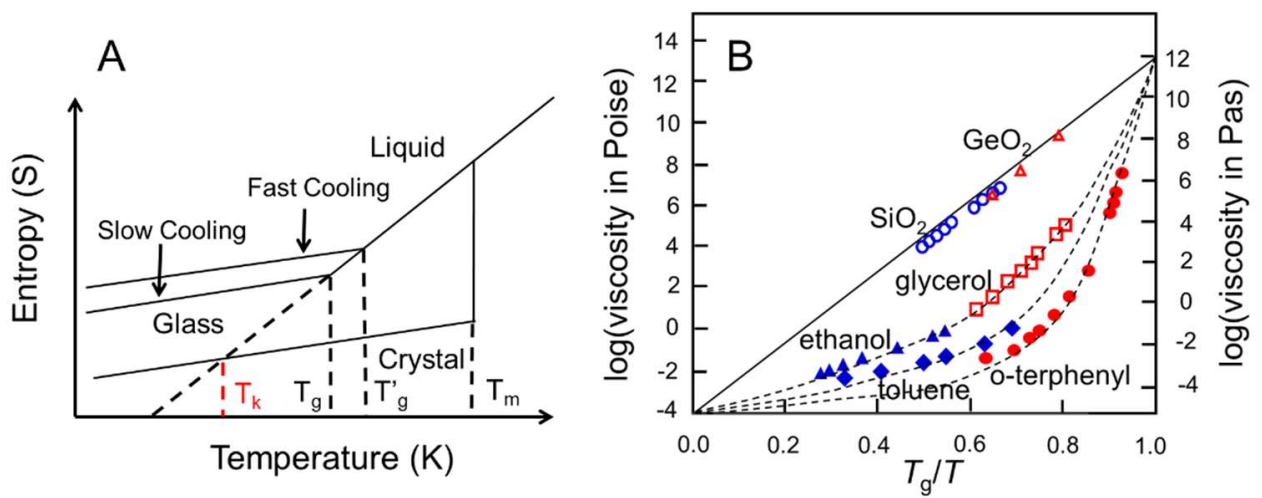

Figure 1. Glass transition fundamentals. (A) Temperature dependent entropy for glass forming materials including monomers and polymers, showing four important temperatures: melting point $T_{\mathrm{m}}$, cooling-rate dependent glass transition temperatures $T_{\mathrm{g}}$ and $T_{\mathrm{g}} \mathrm{g}$, and Kauzmann temperature $T_{\mathrm{k}} ;(\mathbf{B})$ Simplified Angel plot. (A) was adapted from an article (https://www.jps.or.jp/books/gakkaishi/2016/05/71-0570fushigi 09.pdf.) by the Physical Society of Japan. (B) was adapted from ref 2 .

Glass transition similarities in second-order phase transition motivated us to extend our early challenge by fluorescence single-molecule spectroscopy (SMS) and time-resolved spectroscopy using viscosity-sensitive fluorescence probes; ${ }^{5-8}$ and to quest for something unexplored in glass transition. We disclosed two major findings, demonstrating polymer second-order phase transition behavior in a temperature window above $T_{\mathrm{g}}$. One is substantial evidence for poly(vinyl acetate) PVAC collective motion. The other is the discontinuous change in temperature dependent PVAC average relaxation time $\left\langle\tau_{\mathrm{R}}>\right.$ occurring together collective motion enhancement, which looks critical slowing down ${ }^{9}$ in critical phenomena in second-order phase transitions.

\section{RESULTS}

For sample preparation and other technical details, see Materials and Methods in Supporting Information, SI. We used viscosity-sensitive Cy3 in single-molecule spectroscopy (SMS) ${ }^{6}$ due to the fact that its fluorescence quantum efficiency $\Phi_{\mathrm{f}}$ increased with increasing solvent viscosity: $\Phi_{\mathrm{f}}=0.042$ in fluid $(297 \mathrm{~K})$ and 0.94 in rigid $(77 \mathrm{~K})$ ethanol solution, respectively. ${ }^{10}$ We selected PVAC due to its $T_{\mathrm{g}}$ above room temperature $\left(20-25^{\circ} \mathrm{C}\right)$ and below $40{ }^{\circ} \mathrm{C}$ suitable for the present temperature controller. Figure 2A shows the SMS setup we employed in the present work, with which we collected SMS fundamental data (Figures 2B-2F). All data not specified were obtained by the use of PVAC (MW 100,000).

Horizontally polarized excitation (Figure 2A) allowed to collect more single-molecule fluorescence photons compared with vertically polarized excitation due to $\mathrm{Cy} 3$ absorption transition moment being highly oriented on a substrate surface at room temperature $\left(23.8^{\circ} \mathrm{C}\right.$, 1.77-fold, Figures S1A and S1B) and at an elevated temperature $\left(56.3^{\circ} \mathrm{C}, 1.50\right.$-fold, Figures S1C and S1D). One finds more about the polarized excitation and circularly polarized excitation (Figures S2A and S2B) in related descriptions in Supplementary Text in SI.

We applied SMS to the present glass transition-related research using surface-immobilized single molecules. To justify such application, we examined single molecule $\mathrm{Cy} 3$ fluorescence trajectory $I_{\mathrm{f}}(\mathrm{t})$ and autocorrelation functions $C(\tau)$ evaluated from $I_{\mathrm{f}}(\mathrm{t})$. Enhanced fluorescence intensities (2.16-fold, Figures S3A and S3B) with PVAC overlay and no cosine $C(\tau)$ without PVAC overlay (Figures S4A-S4C) support single Cy3 capabilities to detect changes in PVAC environment accompanied by glass transition. One find more about the use of surfaceimmobilized probe molecules in related descriptions in Supplementary Text in SI. 

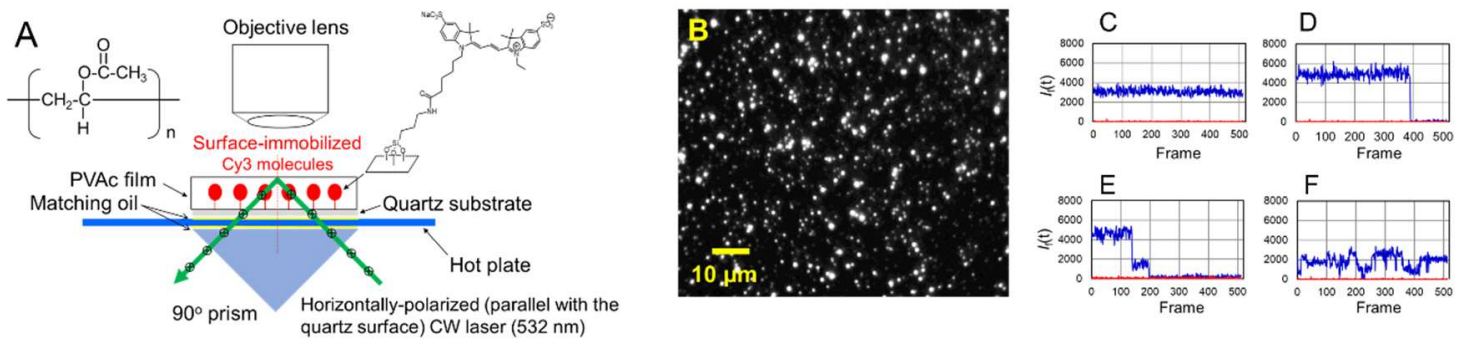

Figure 2. Single-molecule spectroscopy (SMS) fundamentals. (A) Experimental setup for SMS used in the present work: single $\mathrm{Cy} 3$ molecules covalently immobilized on a quartz surface with PVAC overlay, light microscope (shown as objective lens), quartz prism for horizontally polarized (parallel with the sample surface) evanescent illumination, and glass hot plate transparent in the visible region; (B) wide-field fluorescence image averaged over 512 frames ( $36 \mathrm{~ms} /$ frame) followed by background subtraction. Fluorescence trajectory $I_{\mathrm{f}}(t)$ for single $\mathrm{Cy} 3$ molecules at room temperature $\left(24.5^{\circ} \mathrm{C}\right)$ showing $(\mathbf{C})$ no photobleaching, (D) one-step photobleaching, $(\mathbf{E})$ two-step photobleaching, and (F) substantial fluctuation.

\section{Single Cy3 molecule fundamental observations}

Figure 2B shows a single molecule Cy3 fluorescence image with PVAC overlay at $24.5{ }^{\circ} \mathrm{C}$. Averaging 512 frames $(36 \mathrm{~ms} /$ frame) followed by background subtraction generated this image. To evaluate photo-bleached spots in the 512-frame acquisition, we collected one hundred fluorescent spots at $22-25^{\circ} \mathrm{C}: 17-23 \%$ spots, photo-bleached with PVAC overlay; $35-40 \%$ spots, photo-bleached without PVAC overlay; more than $70 \%$ spots, temporally stable with PVAC overlay (Figure 2C). Single staircase photobleaching (Figure 2D) confirmed single molecule observations with rare $(0.1 \%$ maximum) two-step photobleaching occurrences (Figure 2E), showing one brighter fluorescent spot included two Cy3 molecules. We found a few $(\approx 5 \%)$ highly fluctuating spots (Figure 2F).

\section{Temperature dependent single molecule fluorescence trajectories}

We selected $I_{\mathrm{f}}(t)$ 20-30 fluorescent spots that survived from photo-bleaching in 512-frame video acquisition in a pair of heating and cooling experiment at each temperature.

Figures $3 \mathrm{~A}-3 \mathrm{E}$ illustrate representative $I_{\mathrm{f}}(t), C(\tau)$, and power spectrum $J(v)$, Fourier transform of $C(\tau)$, while heating $24.5-64.9^{\circ} \mathrm{C}$. From temperatures elevated in a step-by-step manner: 24.5 , $28.3,29.6,32.0,35.6,37.8,41.9,45.4,51.9,56.4,60.2$, and $64.9^{\circ} \mathrm{C}$ at an average of $0.2 \mathrm{~K} / \mathrm{min}$ (from now on, we omit "an average of" from temperature rate notations in SMS measurements, but not for DSC measurements), these figures include selected temperatures to highlight intermittent $I_{\mathrm{f}}(t)$ fluctuation and marked $C(\tau)$ cosine waveforms at 41.9 and $56.4{ }^{\circ} \mathrm{C}$, and temperature dependent specific sub-second frequencies simultaneously appeared in enhanced and reduced $J(v)$ amplitude: 0,096 at $0.109 \mathrm{~Hz}, 0.472$ at $0.217 \mathrm{~Hz}$, and 0.200 at $0.542 \mathrm{~Hz}$, where the highest $J(v)$ values were recorded at $29.6,41.9$, and $56.4{ }^{\circ} \mathrm{C}$, respectively.

Cosine waveforms in $C(\tau)$, enhanced and reduced $J(v)$ amplitudes, and increasing frequencies in $J(v)$ with increasing temperature prompted us to classify $C(\tau)$. Figure $4 \mathrm{~A}$ and Table S1A summarize occurrences for each $C(\tau)$ type in Figures $3 \mathrm{~A}-3 \mathrm{E}$ at the temperatures specified in these figures. We employed classification criteria for $I_{\mathrm{f}}(t)$ as follows: Type $\mathrm{I}$, stable $I_{\mathrm{f}}(t)$ and no specific peaks in $J(v)$; Type II, fluctuating $I_{\mathrm{f}}(t)$ but no touching the base line with a small $(<0.10)$ single peak in $J(v)$; Type III, fluctuating $I_{\mathrm{f}}(t)$ touching base line with a prominent $(0.20<)$ single peak in $J(v)$; Type IV, fluctuating $I_{\mathrm{f}}(t)$ touching the base line with a prominent $(<0.20)$ single or multiple peaks in $J(v)$; Type $\mathrm{V}$, fluctuating $I_{\mathrm{f}}(t)$ touching the base line without prominent peaks $(0.10<)$ in $J(v)$. One finds similarity between Type $\mathrm{V}$ waveforms $C(\tau)$ and $J(v)$ and those of Type I. We found three or more waveform types at a temperature. For example, $C(\tau) \mathrm{s}$ at $56.4{ }^{\circ} \mathrm{C}$ included Type II, III, IV, and V occurrences (Figure 4A and Table S1A). 

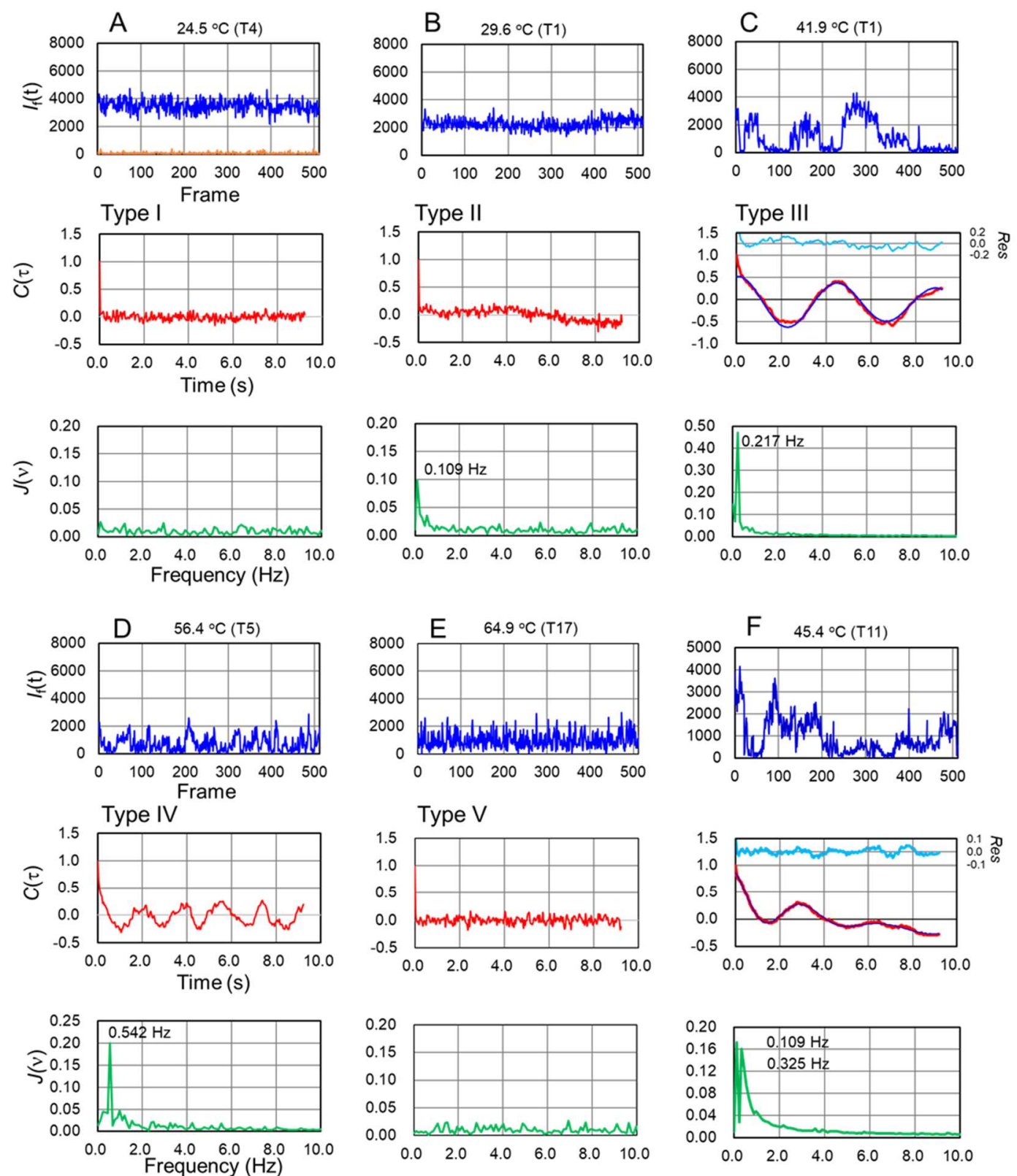

Figure 3. Temperature dependent representative fluorescence trajectories, $I_{\mathrm{f}}(t)$; autocorrelation functions, $\boldsymbol{C}(\boldsymbol{\tau})$; and power spectra, $\boldsymbol{J}(\boldsymbol{v})$. They were observed from single $\mathrm{Cy} 3$ molecules with PVAC overlay from heating experiment $(0.2 \mathrm{~K} / \mathrm{min})$ at (A) 24.5, (B) 29.6, (C) 41.9, (D) 56.4, (E) 64.9, and (F) $45.4{ }^{\circ} \mathrm{C}$. Sets (C) and (F) include the residue (light blue trace) between experimental (red trace) and computed (blue trace) traces. Labels Type I to Type $\mathrm{V}$ are for $C(\tau)$ classification. Video images generating these data were captured with horizontally polarized excitation and averaged over 512 frames ( $36 \mathrm{~ms} /$ frame) followed by background subtraction.

$J(v)$ amplitudes can be a measure of purity in cosine waveforms. We compared the highest $J(v)$ 0.472 at $41.9{ }^{\circ} \mathrm{C}$ with amplitude $0.930(0.977 \mathrm{~Hz})$ computed by Fourier transform of the noisefree and damping-free unitary cosine wave at $1 \mathrm{~Hz}(\cos 2 \pi \mathrm{t})$ with $36 \mathrm{~ms}$ bin time, showing $>50 \%$ $(0.472 / 0.930)$ purity for $C(\tau)$ (Figure 3C).

Figures 3A-3E show representative $J(v)$, with highest amplitudes from 0.096 at $29.6{ }^{\circ} \mathrm{C}$ to 0.200 at $56.4{ }^{\circ} \mathrm{C}$ and maximum overall amplitude 0.472 at $41.9{ }^{\circ} \mathrm{C}$. To make sure that this tendency proves fundamental, we evaluated $C_{\mathrm{av}}(\tau)$ by averaging twenty $C(\tau)$ waveforms survived 
from photobleaching and computed $J_{a v}(v)$ from $C_{\mathrm{av}}(\tau)$ in heating experiment (Figures $5 \mathrm{~A}-5 \mathrm{~L}$ ). The maximum $J_{\mathrm{av}}(v)$ at each temperature showed the highest peak at $41.9^{\circ} \mathrm{C}$.

To avoid possible misunderstandings that all observed $C(\tau)$ continued forever, Figure $3 \mathrm{~F}$ shows damped $C(\tau)$ oscillation classified into Type IV and reproduced by the sum of cosine waveforms multiplied by a single exponential decay amplitude: $C(\tau)=C_{0} \exp \left(-t / C_{1}\right) \times\left\{C_{2} \cos \left(C_{3} t+C_{4}\right)+\right.$ $\left.C_{5} \cos \left(C_{6} t+C_{7}\right)+C_{8} \cos \left(C_{9} t+C_{10}\right)+C_{11} \cos \left(C_{12} t+C_{13}\right)\right\}+C_{14}$, respectively. Type III and IV waveforms commonly included damped $C(\tau)$ oscillation. Figures $3 \mathrm{C}$ includes $C(\tau)$ damped oscillations fitted with $C(\tau)=C_{0} \exp \left(-t / C_{1}\right)\left\{C_{2} \cos \left(C_{3} t+C_{4}\right)\right\}+C_{5}$. In Figure 3F, we used the fitting function including four cosine waveforms against immediate expectation from $J(v)$ : two cosine functions including two distinct frequencies 0.109 and $0.325 \mathrm{~Hz}$ in $J(v)$. Tailing higher than $0.325 \mathrm{~Hz}$ peak likely generated unexpected fact.

In a similar way to Figures $3 \mathrm{~A}-3 \mathrm{~F}$, we present Figures $\mathrm{S} 5 \mathrm{~A}-\mathrm{S} 5 \mathrm{~F}$ to show $I_{\mathrm{f}}(t), C(\tau)$, and $J(v)$ cooling from 64.6 to $25.3{ }^{\circ} \mathrm{C}$ at $0.2-0.3 \mathrm{~K} / \mathrm{min}$. Figure $4 \mathrm{~B}$ and Table S1B summarize Type I to $\mathrm{V}$ occurrences at each temperature for cooling.

Symbols such as T1 and T17 in Figures 3A-3F, S4A-S4C, and S5A-S5F, identify fluorescence spots in the video images mainly for authors' convenience to easily trace highlighted location on the original video images.

A

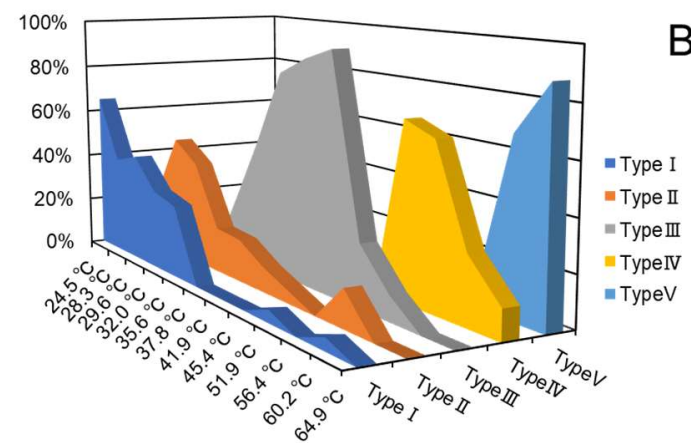

B

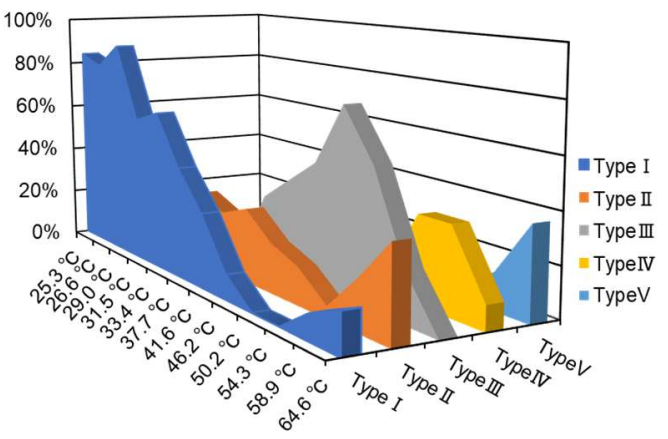

Figure 4. Autocorrelation $\boldsymbol{C}(\tau)$ classification into five types for each observed temperature. Type I to Type V occurrences for (A) heating $24.5-64.9{ }^{\circ} \mathrm{C}(0.2 \mathrm{~K} / \mathrm{min})$ and $(\mathbf{B})$ cooling from $64.6-25.3{ }^{\circ} \mathrm{C}(0.2-0.3 \mathrm{~K} / \mathrm{min})$ are summarized. Photo-bleaching free twenty fluorescent spots from single $\mathrm{Cy} 3$ molecules with PVAC overlay were collected at each temperature. Video images generating these data were captured with horizontally polarized excitation and averaged over 512 frames ( $36 \mathrm{~ms} /$ frame) followed by background subtraction.

\section{Relaxation time evaluation from autocorrelation functions}

Figures $3 \mathrm{C}$ and $3 \mathrm{D}$ present representative $C(\tau)$ showing a cosine waveform and $J(v)$ for heating and Figures S5B and S5C for cooling. However, $J(v)$ was insufficient to evaluate damped $C(\tau)$ oscillations, such as those in Figures $3 \mathrm{~F}$ and S5F; $J(v)$ extracted only oscillatory characteristics from $C(\tau)$. We thus extracted relaxation time $\tau_{\mathrm{R}}$ from $C(\tau)$; however, various waveforms in individual $C(\tau)$ made it difficult to decide fitting functions including several cosine functions like the $C(\tau)$ fitting in Figure 3F. To lift the issue, we used $C_{\text {av }}(\tau)$ to cancel oscillatory characteristics, in particular frequencies higher that $0.1 \mathrm{~Hz}$, from $C(\tau)$. We used the Kohlrausch-Williams-Watts (KWW) function $\exp \left[-\left(t / \tau_{\mathrm{R}}\right)^{\beta}\right]^{1,3}(0<\beta<1)$, which has widely been used for $\alpha$ process relaxation analysis.

We computed $\tau_{\mathrm{R}}$ from $C_{\mathrm{av}}(\tau)$ after converting $C_{\mathrm{av}}(\tau)$ into $C_{\mathrm{av}}(\mathrm{t})$ using

$$
C_{a v}(t)=C_{o} \exp \left[-\left(t / C_{1}\right)^{C_{2}}\right]+C_{3} \cos \left(C_{4} t+C_{5}\right)+C_{6},
$$

where $C_{\mathrm{o}}, C_{1}=\tau_{\mathrm{R}}, C_{2}=\beta, C_{3}, C_{4}, C_{5}$, and $C_{6}$, all fitting parameters. Note that the $C_{\mathrm{av}}(\tau)$ unity at $\tau$ $=0$ was removed from $C_{\mathrm{av}}(\tau)$ to allow $\tau_{\mathrm{R}}$ computation easy; instead, the $C_{\mathrm{av}}(\tau)$ value at $\tau=0.036$ 
s was pasted as the value at $\tau=0$. The KWW function extracts $\tau_{\mathrm{R}}$ distribution in $C_{\mathrm{av}}(\tau)$ through the parameter $\beta$. The evaluated $C_{1}=\tau_{\mathrm{R}}$ is then converted into average relaxation time $<\tau_{\mathrm{R}}>$ using

$$
\left\langle\tau_{R}\right\rangle=\int_{0}^{\infty} e^{-\left(t / \tau_{R}\right)^{\beta}} d t=\left(\tau_{R} / \beta\right) \Gamma(1 / \beta),
$$

where $\Gamma$, the gamma function; and the area under the KWW function in eq $3,<\tau_{R}>$. ${ }^{11}$ Figures $5 \mathrm{~A}-$ $5 \mathrm{~L}$ show all the temperature dependent $C_{\mathrm{av}}(\tau),\left\langle\tau_{\mathrm{R}}>\right.$, and $J_{\mathrm{av}}(v)$, Fourier transform of $C_{\mathrm{av}}(\tau)$. The computed $\beta$ ranged 0.45 to 1.0 and 0.36 to 1.0 for heating in Figures $5 \mathrm{~A}-5 \mathrm{~L}$ and for cooling in Figure S6A-S6L, respectively. In the subsequent section, we employ $J_{\mathrm{av}}(v)$ to find specific temperature windows showing the maximum $J_{\mathrm{av}}(v)$ intensities in heating and cooling experiments.

\section{Temperature dependent relaxation times in a specific temperature window near $\boldsymbol{T}_{\mathrm{g}}$}

We compared DSC measurements, temperature dependent $J_{\mathrm{av}}{ }^{\max }$, which means $J_{\mathrm{av}}(v)$ maxima at each temperature, and $\left\langle\tau_{\mathrm{R}}>\right.$ to find a temperature window, in which each $J_{\mathrm{av}}{ }^{\max }$ and $<\tau_{\mathrm{R}}>$ peak was identified. Figures $6 \mathrm{~A}$ and $6 \mathrm{~B}$ show PVAC DSC measurements for heating and cooling both at $5 \mathrm{~K} / \mathrm{min}$, respectively, in which we noted three specific temperatures: $T_{1}=33.0{ }^{\circ} \mathrm{C}$ (heating), $T_{2}=43.2{ }^{\circ} \mathrm{C}$ (cooling), and $T_{3}=30.4{ }^{\circ} \mathrm{C}$ (cooling). In a cooling experiment, we evaluated $T_{\mathrm{g}}=$ $36.5^{\circ} \mathrm{C}(309.5 \mathrm{~K})$ from the midpoint of the gap between extrapolated glass and melt lines. In addition to the experiment giving Figures $6 \mathrm{~A}$ and $6 \mathrm{~B}$, we evaluated $T_{\mathrm{g}}$ by cooling from a series of heating, cooling, and again heating DSC measurements (Figures S7A-S7C) at three temperature rates, $5,10,20 \mathrm{~K} / \mathrm{min}$. Only cooling experiment presented consistent results showing 36.0$36.3{ }^{\circ} \mathrm{C}$ as $T_{\mathrm{g}}$ from the midpoint evaluation irrespective of the different temperature rates, confirming that cooling from melt erases the inhomogeneous glass structure memory.

Figure 6A shows another temperature zone under the dotted line above $T_{1}=33.0{ }^{\circ} \mathrm{C}$ characterized by enthalpy overshoot, ${ }^{12}$ which characterizes an endothermic process intrinsic to heating DSC. This overshoot always occurred in our observations (Figure 6A and Figures S7AS7C). In contrast, we observed no enthalpy overshoots for cooling experiments (Figure 6B and Figures S7A-S7C). Note that the heating DSC trace in Figure 6A totally differs from the heating traces in Figures S7A-S7C, which shows enthalpy overshoot much larger than that in Figure 6A. The difference reflects difficulties in getting reproducible heating DSC traces.

The same SMS movie at each temperature generated a set of temperature dependent $J_{\mathrm{av}}{ }^{\max }$ and $<\tau_{\mathrm{R}}>$ as assembled in Figures $6 \mathrm{C}$ and $6 \mathrm{E}$; alike Figures $6 \mathrm{D}$ and $6 \mathrm{~F}$, Figures $6 \mathrm{G}$ and 6I, and Figures $6 \mathrm{H}$ and $6 \mathrm{~J}$. Heating and cooling experiments were carried out using independent samples from the same preparation lot.

To compare the temperature dependent $\left\langle\tau_{\mathrm{R}}>\right.$ with temperature dependent relaxation times evaluated by dielectric loss spectroscopy, we computed VFT lines using another VFT equation

$$
\log \left(\omega_{\infty} / \omega_{\max }\right)=B /\left(T-T_{o}\right),
$$

where $\omega_{\infty}$, the limiting angular velocity at infinite temperature. Using $B=660 \pm 50 \mathrm{~K}^{13}$ in addition to other three $B$ values we selected, and then converting $\omega_{\max }=2 \pi v$ into $\left\langle\tau_{\mathrm{R}}>\right.$ from $1 / v=\left\langle\tau_{\mathrm{R}}>\right.$, we computed VFT lines and superposed them on Figures 6E, 6F, 6I, and 6J, showing that enhanced $<\tau_{\mathrm{R}}>$ substantially followed the VFT lines. 

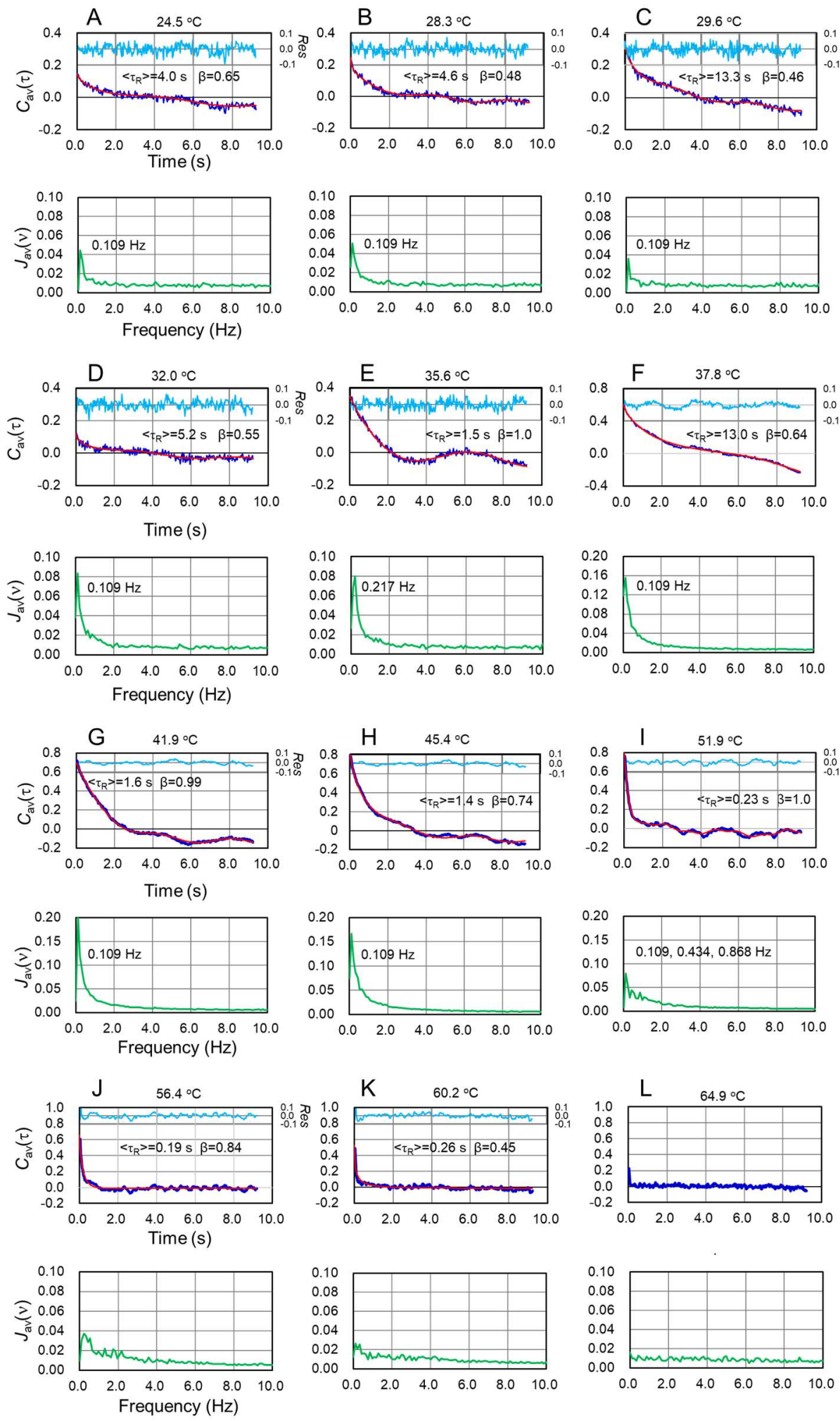

Figure 5. Average autocorrelation functions, $\boldsymbol{C}_{\mathrm{av}}(\tau)$ and power spectra, $\boldsymbol{J}_{\mathrm{av}}(v)$. They were observed for heating $(0.2 \mathrm{~K} / \mathrm{min})$ from single $\mathrm{Cy} 3$ molecules with PVAC overlay and computed from twenty single Cy3 molecules free from photobleaching at (A) 24.5, (B) 28.3, (C) 29.6, (D) 32.0, (E) 35.6, (F) 37.8, (G) 41.9, (H) 45.4, (I) 51.9 , (J) $56.4,(\mathbf{K}) 60.2$, and (L) $64.9^{\circ} \mathrm{C}$. In (L), $<\tau_{\mathrm{R}}>$ evaluation was halted due to inability to identify decay in $C_{\text {av }}(\tau)$. Each $C_{\text {av }}(\tau)$ trace except for $(\mathbf{L})$ was fitted with eq 2 and includes calculated trace (red) and parameters $<\tau_{\mathrm{R}}>$ and $\beta$, together with residue between experimental and calculated traces (light blue). 

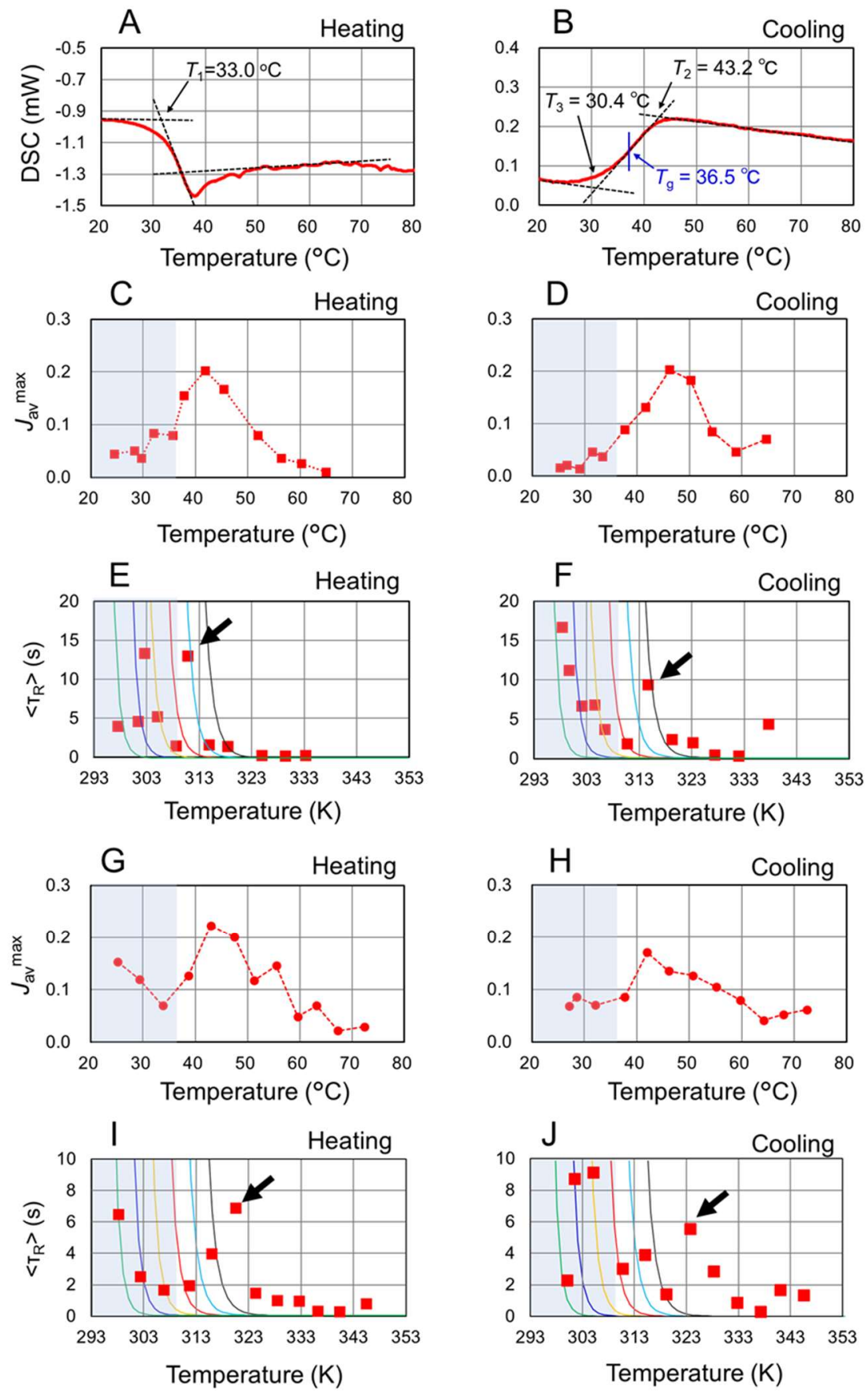

Figure 6. DSC measurements; temperature dependent average power spectra maxima, $J_{a v}{ }^{\text {max }}$; and temperature dependent average relaxation time, $<\tau_{\mathrm{R}}>$ superposed with VFT lines: DSC traces for (A) heating and (B) cooling (both $5.0 \mathrm{~K} / \mathrm{min}) ; J_{\mathrm{av}}{ }^{\max }$ for $(\mathbf{C})$ heating $(0.2 \mathrm{~K} / \mathrm{min})$ and $(\mathbf{D})$ cooling $(0.2-0.3 \mathrm{~K} / \mathrm{min}) ;<\tau_{\mathrm{R}}>$ for (E) heating and (F) cooling $(0.2-0.3 \mathrm{~K} / \mathrm{min})$. VFT lines $(B=460,510,560,610,660$, and $710 \mathrm{~K}$ in green, blue, orange, red, light blue, and gray, respectively, computed from eq 4 using each $B$ value) are superposed on the $<\tau_{\mathrm{R}}>$ plot. From another pair of heating and cooling (both $1 \mathrm{~K} / \mathrm{min}$ ) experiments using PVAC (MW 500,000), we obtained data in $(\mathbf{G}),(\mathbf{H}),(\mathbf{I})$ and $(\mathbf{J})$. The VFT lines for $B=610,660$, and $710 \mathrm{~K}$ were experimentally identified. ${ }^{13}$ Arrows in $(\mathbf{E}),(\mathbf{F}),(\mathbf{I})$, and $(\mathbf{J})$ specify discontinuously enhanced $<\tau_{\mathrm{R}}>$ peaks. The temperature zones in a shaded blue indicate those below $T_{\mathrm{g}}=36.5^{\circ} \mathrm{C}$. The standard deviation $\pm \sigma$ of each data point in (E) was within the marker size except for $9.96 \mathrm{~s}$ at $302.6 \mathrm{~K}$. In $(\mathbf{F}), 94.3 \mathrm{~s}, 12.1 \mathrm{~s}, 7.0 \mathrm{~s}, 3.4 \mathrm{~s}, 2.1 \mathrm{~s}, 6.5 \mathrm{~s}$, and $3.6 \mathrm{~s}$ are beyond the marker size at $298.3 \mathrm{~K}, 299.6 \mathrm{~K}, 302.0 \mathrm{~K}, 304.5 \mathrm{~K}, 306.4 \mathrm{~K}, 314.6 \mathrm{~K}$, and $337.6 \mathrm{~K}$, respectively. In $(\mathbf{I})$, all $\pm \sigma$ for $<\tau_{\mathrm{R}}>$ was within the marker size. In $(\mathbf{J}), 1.31 \mathrm{~s}, 8.18 \mathrm{~s}$, and $1.68 \mathrm{~s}$ are beyond the marker size at $301.5 \mathrm{~K}, 305.1 \mathrm{~K}$, and $332.7 \mathrm{~K}$, respectively. 


\section{DISCUSSION}

To understand the significance of two major findings, we first summarize similarity and difference between glass transition and critical phenomena in second-order phase transitions; second, remind us of glass transition basics from dielectric spectroscopy to reveal significance and to find technical limitations in the present work; third, provide supplementary views about each experimental observation and substantial consideration to observations related to the two major findings; last, develop arguments to help understand the two major findings.

\section{Glass transition versus critical phenomena in second-order phase transitions}

Non-Arrhenius profiles in Figure 1B suggest one of critical phenomena in second order phase transitions, or collective or cooperative molecular motion. ${ }^{4}$ This process is assigned to $\alpha$ process for glass forming materials. Surrounding molecules form an energy barrier that a molecule of interest must overcome to move away. This barrier increases with lowering temperature, suggesting the surrounding molecules behave collectively to arrest molecule movement. Distinct spatial domains develop along with $\alpha$ process slowdown, generating spatial heterogeneity in glass forming materials. Cooperatively rearranging region $(\mathrm{CRR})^{3}$ materializes such heterogeneity near $T_{\mathrm{g}}$ for many glass forming materials, the size of which is denoted by characteristic length $\xi_{\alpha}$, average CRR diameter. In fact, no one found $\xi_{\alpha}$ divergence in any glass-forming materials. The divergence should generate turbid appearance at $T_{\mathrm{g}}$; however, this is not the case.

In contrast to $\alpha$ process slowing down and CRR extension with decreasing temperature, fluid critical phenomena near $T_{\mathrm{c}}$ exhibited correlation length $\xi$ divergence along with activated collective molecular motion. Critical opalescence in $\mathrm{CO}_{2}$ exemplifies these phenomena, ${ }^{4}$ generating a change in fluid appearance from transparent to turbid and density fluctuations detectable by light scattering measurement and much slower than $\mathrm{CO}_{2}$ molecular motions near $T_{\mathrm{c}}$ known as critical slowing down. Turbid appearance means that $\mathrm{CO}_{2}$ molecules behave collectively in the size beyond or close to visible light wavelengths $400-700 \mathrm{~nm}$. However, no $\xi_{\alpha}$ divergence like critical opalescence occurs along with $\alpha$ process slowing down. This fact sharply discriminate glass transition and critical phenomena. We know similarity between $\alpha$ process and critical phenomena simply through "slowing down".

\section{Glass transition basics from dielectric spectroscopy}

Cooling glass forming liquids below $T_{\mathrm{m}}$ and close to the crossover temperature $T_{\text {cross }} \approx 1.2 T_{\mathrm{g}}$, consistent with mode-coupling theory (MCT) expectation, ${ }^{1,14}$ splits a single dielectric-loss peak into faster and slower ones. ${ }^{1}$ The slower frequency dynamics characterizes $\alpha$ process and the faster frequency does $\beta$ process. Alpha process exhibits non-Arrhenius behavior and almost disappears below $T_{\mathrm{g}}$, whereas $\beta$ process continues Arrhenius behavior below $T_{\mathrm{g}}$. The $\tau_{\mathrm{R}}$ for $\alpha$ process, typically $0.1-1.0 \mathrm{~s}$, at $T_{\mathrm{g}}+10-20 \mathrm{~K}$, presents much slower $\left(>10^{7}\right.$-fold) $\tau_{\mathrm{R}}$ than that for $\beta$ process at $T_{\mathrm{g} .}{ }^{1,3}$ Our SMS technique lacked temporal resolution to detect $\beta$ process; we only considered $\alpha$ process assigned to polymer main chain segment Brownian motion (micro-Brownian motion) characterized by cooperative nature. The size of cooperative regions, or CRR, was estimated at 1-5 nm. ${ }^{1,15-17}$ near $T_{\mathrm{g}}$ giving heterogeneity in many glass forming materials.

\section{Supplementary views about each experimental observation}

First, fluorescence intensity varied from spot to spot (Figure 2B). This was partly due to excitation light polarization and photo-bleached spots in the image acquisition, but mainly due to highly heterogeneous polymer structures. Second, we should follow the identical single Cy3 molecules from start to finish in all SMS measurement; however, photobleaching prohibited such ideal measurement, limiting prolonged SMS experiment. Third, we ascertained the validity of single Cy3 molecules sensing PVAC environment (Figures S3A and S3B; Figures S4A-S4C), and then discussed glass transition at interfaces and sub-second $J(v)$ frequency origins in Figures 3B3D, 3F, S5A-S5D, and S5F in Supplementary Text in SI. Figures S9A-S9D in Supplementary Text in $S I$ summarizes a proposed scheme to explain intermittent $I_{\mathrm{f}}(t)$ fluctuation (Figures $3 \mathrm{C}-3 \mathrm{~F}$ ) as well as Cy3 photophysics behind $I_{\mathrm{f}}(t)$ fluctuation. Last, we used the product of one single 
exponential function and one cosine function or cosine function summation to treat $I_{\mathrm{f}}(t)$ as a single damped oscillator in a conventional manner (Figures $3 \mathrm{~F}$ and S5F). We however employed the sum of the KWW and a cosine function (eq 2) to reproduce experimental observations (Figures $5 \mathrm{~A}-5 \mathrm{~L}$ and Figures S6A-S6L) simply because the product did not generate acceptable fitting between $C_{\mathrm{av}}(\tau)$ and the calculated one, such as no convergence fitting to begin with, negative $C_{\mathrm{o}}$, and $C_{2}$ larger than 1.0 in eq 2 . From eq 2 we extracted only the relaxation term and evaluated $<\tau_{\mathrm{R}}>$ through eq 3 . This procedure follows fluctuation-dispersion theorem.

\section{Substantial consideration to observations related to the two major findings}

(i) Temperature dependent $C(\tau)$ evaluated from $I_{\mathrm{f}}(t)$ (Figures $3 \mathrm{~A}-3 \mathrm{~F}$ for heating and Figures S5A-S5F for cooling) included marked $C(\tau)$ cosine waveforms, hence providing evidence for collective PVAC motion above $T_{\mathrm{g}}$. This indicates the first major finding, PVAC collective motion deduced from the generalized Langevin equation (GLE) ${ }^{18}$ under the approximation leading to the understanding that PVAC environment coherently and persistently influences viscosity sensitive single Cy3 molecule fluorescence.

(ii) Our consideration to the observations in Figures $6 \mathrm{~A}-6 \mathrm{~J}$ depends on the assumption that $T_{\mathrm{g}}$ at interfaces between PVAC films and quartz surfaces $\left(T_{\mathrm{g}}{ }^{\text {int }}\right)$ approximates $T_{\mathrm{g}}$ evaluated by DSC. One finds more about this assumption in Supplementary Text in SI.

(iii) From $\left\langle\tau_{R}>\right.$ evaluation, we found the second major finding, discontinuous $\left\langle\tau_{R}>\right.$ change in a specific temperature window, in which PVAC collective motion was activated. From the observations in Figures $6 \mathrm{C}-6 \mathrm{~J}$, we can mention the fact that $\left\langle\tau_{\mathrm{R}}>\right.$ peaks occurred in the same temperature window where enhanced $J_{\mathrm{av}}{ }^{\max }$ values were observed. Increased viscosity at lower temperatures and Cy 3 viscosity-sensitive nature both justify enhanced $<\tau_{R}>$ with decreasing temperature below $T_{\mathrm{g}}=36.5^{\circ} \mathrm{C}$. From the VFT line superposition (Figures 6E, 6F, 6I, and 6J), the $<\tau_{\mathrm{R}}>$ peaks presented unexpected observations.

\section{Derivation of $\boldsymbol{C}(\tau)$ cosine waveforms from generalized Langevin equation}

The normalized autocorrelation function $C(\tau)$ takes the form of

$$
C(\tau)=R(\tau) / R(0),
$$

where $R(\tau)$, the autocorrelation function; and $R(0)$, a normalizing factor. Before evaluating $C(\tau)$ we computed $R(\tau)$ from $I_{\mathrm{f}}(t)$ :

$$
R(\tau)=\frac{1}{N} \sum_{i}^{N} I_{f}^{o}(i) I_{f}^{o}(i+\tau) \equiv\left\langle I_{f}^{o}(i) I_{f}^{o}(i+\tau)\right\rangle,
$$

where $I_{\mathrm{f}}^{\circ}(i)=I_{\mathrm{f}}(i)-\bar{I}_{\mathrm{f}} ; \bar{I}_{\mathrm{f}}$, average $I_{\mathrm{f}}(t)$ over 512 frames; $N=256$, the number of data points (half the number of the total frames) with step size or bin time $\Delta t=36 \mathrm{~ms}$; and $i$ and $\tau$, each data point.

We summarize cosine autocorrelation function derivation from the GLE ${ }^{19}$

$$
\frac{d A(t)}{d t}=i \omega_{o} A(t)-\int_{0}^{t} \varphi\left(t-t^{\prime}\right) A\left(t^{\prime}\right) d t^{\prime}+f(t),
$$

where $A(\mathrm{t})$, a dynamical variable (it is a vector in a general form); $\omega_{0}$, a formal angular frequency (it is a matrix with zero diagonal elements in a general form); $f(\mathrm{t})$, a randomly fluctuating external force; and $\varphi\left(t-t^{\prime}\right)$, a memory function combining $A(\mathrm{t})$ at $t^{\prime}$ (past) and $t$ (present) given by

$$
\varphi\left(t-t^{\prime}\right)=\frac{\left\langle f(t) f\left(t^{\prime}\right)\right\rangle}{\langle f(0) f(0)\rangle},
$$

Equation 8 establishes an essential result from GLE formulation, ${ }^{18}$ signifying that the memory function explicitly combines with normalized external force time correlation function. This equation belongs to fluctuation dispersion theorem. For the present derivation, $A(\mathrm{t})$ is a scaler variable equivalent to $I_{\mathrm{f}}(t)$; hence $\omega_{\mathrm{o}}=0$ because it is a $[1 \times 1]$ dimensional matrix with zero diagonal. 
We applied GLE to Brownian motion analysis, and it has reason for the use here considering that polymer segment micro-Brownian motion models polymer dynamics. After several derivation steps from eq 7 (Supplementary Text in SI includes the skipped derivations),

$$
\frac{d \Phi(t)}{d t}=-\omega^{2} \int_{0}^{t} \varphi\left(t-t^{\prime}\right) \Phi\left(t^{\prime}\right) d t^{\prime},
$$

where $\omega^{2}$, constant; and $\Phi(\mathrm{t})$, a normalized autocorrelation function equivalent to $C(\tau)$,

$$
\Phi(t)=\frac{\langle A(t) A(0)\rangle}{\langle A(0) A(0)\rangle},
$$

where $<A(\mathrm{t}) A(0)>$, an autocorrelation function of $A(\mathrm{t})$. Substituting $\varphi\left(t-t^{\prime}\right)=1$ into eq 9 , no memory function extinction approximation, ${ }^{19}$ gives

$$
\frac{d \Phi(t)}{d t}=-\omega^{2} \int_{0}^{t} \Phi\left(t^{\prime}\right) d t^{\prime}
$$

and hence

$$
\Phi(t)=\cos \omega t
$$

because $\Phi(0)=1$ from the normalized autocorrelation function definition. We thus obtain a cosine autocorrelation function from no extinction approximation to memory function. This provides the rationale for cosine $C(\tau)$ waveforms (Figures $3 \mathrm{C}$ and $\mathrm{S} 5 \mathrm{C}$ ) and can be explained as follows.

No memory function extinction $\left\langle f(t) f\left(t^{\prime}\right)>\approx \varphi\left(t-t^{\prime}\right)=\right.$ constant means PVAC collective motion coherently and persistently affecting single Cy3 molecules as Brownian particles. On the other hand, instantaneous $\varphi\left(t-t^{\prime}\right)$ disappearance approximation provides a single exponential decay $C(\tau)$. Damped oscillation (Figures $3 \mathrm{~F}$ and S5F) as a combined waveform cosine and single exponential decay dominated many experimental observations. Figures S10A and S10B compare the two approximations for GLE in SI.

\section{Essentials in heating, cooling, and different PVAC molecular weight experiments}

Two $J_{\text {av }}{ }^{\max }$ peaks at 41.9 (Figure $6 \mathrm{C}$, heating) and $46.2{ }^{\circ} \mathrm{C}$ (Figure 6D, cooling) showed separation by $\approx 4 \mathrm{~K}$. This appears hysteresis between heating and cooling. Another pair of heating and cooling experiments using PVAC (MW 500,000), Figure 6G (heating at $1 \mathrm{~K} / \mathrm{min}$ ) and Figure $6 \mathrm{H}$ (cooling at $1 \mathrm{~K} / \mathrm{min}$ ), exhibited no substantial hysteresis. Such small hysteresis varied from experiment to experiment in an uncontrollable manner, independent of MW 100,000 or 500,000 and heating and cooling rate $(1 \mathrm{~K} / \mathrm{min}$ or slower); the different hysteresis observations cannot be ascribed to the MW and temperature rate difference. Importance lies in the fact that heating and cooling experiments identified $J_{\mathrm{av}}{ }^{\max }$ reaching the top between $T_{\mathrm{g}}$ and nearly $T_{\mathrm{g}}+10 \mathrm{~K}$.

In the comparison between temperature dependent $<\tau_{\mathrm{R}}>$ in a heating and cooling pair in Figures 6E and 6F and another pair in Figures 6I and 6J using PVAC (MW 500,000), we experienced the comparison similar to that between heating $J_{\mathrm{av}}{ }^{\max }$ and cooling $J_{\mathrm{av}}{ }^{\max }$ peaks. Again, importance lies in the fact that $\left\langle\tau_{\mathrm{R}}>\right.$ reached the top pointed by arrows between $T_{\mathrm{g}}$ and nearly $T_{\mathrm{g}}+10 \mathrm{~K}$ independent of MW. This mention sounds reasonable considering that PVAC (MW 500,000) $T_{\mathrm{g}}$ was almost equal to that of PVAC (MW 100,000) (Figures S8A-S8C).

\section{Discontinuous change in temperature-dependent relaxation time above $\boldsymbol{T}_{\mathrm{g}}$}

Temperature dependent $J_{\mathrm{av}}{ }^{\max }$ (Figures $6 \mathrm{C}, 6 \mathrm{D}, 6 \mathrm{G}$, and $6 \mathrm{H}$ ) disclosed a temperature window between $T_{\mathrm{g}}=36.5^{\circ} \mathrm{C}(309.5 \mathrm{~K})$ and $T_{\mathrm{g}}+10-20 \mathrm{~K}$. Temperature dependent $<\tau_{\mathrm{R}}>$ (Figures $6 \mathrm{E}, 6 \mathrm{~F}$, $6 \mathrm{I}$, and $6 \mathrm{~J}$ ) pinpointed specific temperatures within the temperature window, showing the second major finding. These temperatures fall below $T_{\text {cross }} \approx 1.2 T_{\mathrm{g}}=371.4 \mathrm{~K}\left(98.4{ }^{\circ} \mathrm{C}\right)$. Thus, the temperature window characterized by highly fluctuating collective PVAC motions embodied by the $C(\tau)$ cosine waveform appears outside MCT expectation but involves temperature zone showing cooperative $\alpha$ process activity.

We examine the significance of $<\tau_{\mathrm{R}}>$ discontinuous change generating peaks (Figures $6 \mathrm{E}, 6 \mathrm{~F}$, 
$6 \mathrm{I}$, and $6 \mathrm{~J})$. These temperatures fall within the temperature window where $J_{\mathrm{av}}{ }^{\max }$ was enhanced (Figures $6 \mathrm{C}, 6 \mathrm{D}, 6 \mathrm{G}$, and $6 \mathrm{H}$ ). Higher $J_{\mathrm{av}}{ }^{\max }$ amplitude was associated with activated $C_{\mathrm{av}}(\tau)$ cosine or oscillatory characteristics (Figures $5 \mathrm{~A}-5 \mathrm{~K}$ and S6A-S6L). We thus consider the temperatures giving $\left\langle\tau_{\mathrm{R}}>\right.$ peaks to be $T_{\mathrm{c}}$ (heating) and $T_{\mathrm{c}}$ ' (cooling) from the following discussion.

Ideal cosine $C(\tau)$ without damping shows infinite $\tau_{\mathrm{R}}$. In practice, $<\tau_{\mathrm{R}}>$ exhibited limited-height peaks within the temperature window between $T_{\mathrm{g}}=36.5^{\circ} \mathrm{C}(309.5 \mathrm{~K})$ and $T_{\mathrm{g}}+10-20 \mathrm{~K}$, in which one finds $C_{\mathrm{av}}(\tau)$ cosine characteristics, or collective PVAC motion activation. The $<\tau_{\mathrm{R}}>$ peaks look equivalent to order parameter discontinuity in second-order phase transitions or critical phenomena ${ }^{4}$ because of their simultaneous occurrences with activated collective PVAC motion. Thus, $<\tau_{\mathrm{R}}>$ discontinuity associated with PVAC collective motion reminds us of critical slowing down, which is a critical phenomenon in second-order phase transitions.

Critical slowing down ${ }^{9}$ provides divergent $\tau_{\mathrm{R}}$ near $T_{\mathrm{c}}$

$$
\tau_{R} \propto \frac{1}{\left(T-T_{c}\right)^{\gamma}},
$$

where $\gamma$, a critical-point exponent. A moving particle on a potential energy curve models critical slowing down as follows. ${ }^{20}$ Consider a particle on the Gibbs potential designated by pressure $p$ and $T$ having two minima. Below $T_{\mathrm{c}}$ changing $p$ or $T$ allows the particle to move from one higher minimum to the lower one, generating the first-order phase transition. At $T_{\mathrm{c}}$ the second-order phase transition occurs; two potential minima merge into one minimum, and the one-minimum potential takes a flattened shape formulated by Landau theory. The particle moves slowly and widely on the flattened potential surface. The slowly moving particle models critical slowing down.

Equation 13 equals temperature dependent isothermal magnetic susceptibility $\chi_{\mathrm{T}}$ in magnets and isothermal compressibility $\kappa_{\mathrm{T}}$ in fluids in divergence at $T_{\mathrm{c}}$. The $\gamma$ value takes unity from the mean-field theory and Landau theory, but experimentally $1.2-1.4 .{ }^{4,9,20}$ We restrict serious $\gamma$ value evaluation from Figures $6 \mathrm{E}, 6 \mathrm{~F}, 6 \mathrm{I}$, and $6 \mathrm{~J}$; however, $\gamma=0.5$ was unrealistic but $\gamma=1$ was possible, and discrimination between $\gamma=1$ and $\gamma=1.5$ was hard to do in the limited number of data points in the tentative evaluation from these figures. The present work mission does not involve rigorous $\gamma$ evaluation but SMS application to glass transition issue elucidation. Experimental challenges to estimate more rigorously $\gamma$ values are now underway. In any way, second-order phase transition behavior was observed not only in cosine $C(\tau)$ but also in temperature dependent discontinuous $<\tau_{R}>$ changes.

Several pairs of heating and cooling SMS experiments reproduced discontinuous change in $<\tau_{\mathrm{R}}>$; however, the temperatures showing the peak $\left\langle\tau_{\mathrm{R}}>\right.$ were different from heating to cooling and experiment to experiment. The varied $\left\langle\tau_{R}>\right.$ peak occurrences can be rationalized as follows. In the present SMS, we employed surface immobilized sub-nanometer size Cy3 probes allowing spatially resolved measurement in the nanometer region. We thus assume that not all the probed sites in PVAC present second-order phase transition behavior. For example, $C(\tau)$ in Type III did not exclusively occur between $T_{\mathrm{g}}=36.5^{\circ} \mathrm{C}(309.5 \mathrm{~K})$ and $T_{\mathrm{g}}+10-20 \mathrm{~K}$ window especially at $T_{\mathrm{g}}+10 \mathrm{~K}$ (Figures 4A and 4B; Tables S1A and S1B). Note that $<\tau_{\mathrm{R}}>$ was evaluated from $C_{\mathrm{av}}(\tau)$ averaged over every $C(\tau)$ including Type I to Type $\mathrm{V}$ at each temperature, likely generating varied $<\tau_{R}>$ peaks.

An early model, ${ }^{21}$ in which super cooled liquids include dispersed micro-crystals, inspires the idea of specific sites showing second-order phase transition behavior in PVAC. This model predicts the widely known empirical relationship $T_{\mathrm{g}} / T_{\mathrm{m}} \simeq 2 / 3$, making this model believable. For this reason, our assumption considering the sites specific to second-order phase transition behavior in PVAC seems to be far from groundless. The idea of super cooled liquids including dispersed micro-crystals have developed into super cooled liquids as mosaic states modeled in random first-order transition (RFOT) theory. ${ }^{22}$ Single Cy3 molecules might probe such mosaic 
state dynamics in PVAC and disclosed its heterogeneity showing second-order phase transition behavior. We have no scientific idea at this moment to replace "first-order" in RFOT with "second-order" based on the present achievements. Instead, the present work starting from phenomenology in thermodynamic and transport dynamic showing similarities in second-order phase transitions in Figures 1A and 1B makes it possible to propose an assumed "random secondorder transition theory" as a game of word substitution.

\section{CONCLUSIONS}

We identified two sets evidence for second-order phase transition behavior in PVAC glass transition above $T_{\mathrm{g}}$ : highly active PVAC collective motions from $C(\tau)$ cosine waveforms and discontinuous transition in temperature dependent $\left\langle\tau_{R}\right\rangle$. These findings appeared beyond the known theoretical framework for glass transition and related phenomena, such as MCT and RFOT.

Second-order phase transition behavior in glass transition has been suggested in temperature dependent $S, C_{\mathrm{p}}$, and $\eta$ as noted in Introduction. These parameters temperature dependence provide solid experimental facts. However, such observations have lacked straightforward evidence for critical phenomena hidden behind glass transition mainly due to their ensemble averaged nature. The major findings arose from SMS, making it possible to directly detect polymer segment micro-Brownian motion. However, SMS in the present our status has one inability and one technical limitation in return for the uniqueness demonstrated in the present work. The inability includes no direct $\xi_{\alpha}$ evaluation. No critical opalescence observation from PVAC suggests $\xi_{\alpha}<100 \mathrm{~nm}$. Photobleaching in Cy3, or most fluorescent dyes, limits SMS usefulness. This issue prevents repeated video imaging, limiting the number of $\left\langle\tau_{R}>\right.$ data points.

\section{ASSOCIATED CONTENT \\ Corresponding Author \\ *E-mail: mi195526@josai.ac.jp \\ ORCID}

Mitsuru Ishikawa: 0000-0001-9438-2270

Takayuki Uwada: 0000-0002-4272-7964

Funding: This work was supported by graduate and undergraduate student research budgets in Department of Chemistry, Josai University; no other funding was involved such as Kaken-hi (MEXT) in the present work. Author contributions: M. I. planned this work, supervised three students, T. T., Y. H., and M.A. and wrote the manuscript. T. T. joined this work for three years as an undergraduate and a graduate student, contributing to experiment and data analysis. Y. H. and M. A. also joined this work for one year each as undergraduate students and contributed to part of experiment and data analysis. T. U. also supervised T. T. to complete his master's thesis involving this work. Competing interests: All the authors have no competing interests. Data and material availability: All data necessary for evaluating the conclusions in the present work are included in the manuscript and SI.

\section{ACKNOWLEDGMENTS}

M. I. thanks Prof. Vasudevan Pillai Biju, Research Institute for Electronic Science, Hokkaido University, for his kind advice and support for sample preparation, and also for permitting the use of Figures S9A-S9C, the original of which was prepared by himself. Also, Prof. Yoshihiro Tokudome, Department of Pharmaceutical Sciences, Josai University, is appreciated by M. I. for his technical advice for DSC equipment operation in 2019. 


\section{REFERENCES}

1. P. G. Debenedetti, F. H. Stillinger, Supercooled liquids and the glass transition. Nature 410, 259-267 (2001).

2. C. A. Angell, K. L. Ngai, G. B. McKenna, P. F. McMillan, S. W. Martin, Relaxation in glassforming liquids and amorphous solids. J. Appl. Phys. 88, 3113-3157 (2000).

3. E. Donth, "1. Introduction" and "2.1. The classical picture. No serious problems" in The Glass Transition, Relaxation Dynamics in Liquid and Disordered Materials, (Springer 2001), pp 1-66.

4. H. E. Stanley, "What are the critical phenomena? A survey of some basic results" in Introduction to phase transitions and critical phenomena, (Oxford 1971), pp. 1-21.

5. M. Ishikawa, J. Y. Ye, Y. Maruyama, H. Nakatsuka, Triphenylmethane dyes revealing heterogeneity of their nanoenvironment: femtosecond, picosecond, and single-molecule studies. J. Phys. Chem. A 103, 4319-4331 (1999).

6. V. P. Biju, J. Y. Ye, M. Ishikawa, Spatial heterogeneity in a polymer thin film probed by single molecules. J. Phys. Chem. B 107, 10729-10735 (2003).

7. J. Y. Ye, T. Hattori, H. Inouye, H. Ueta, H. Nakatsuka, Y. Maruyama, M. Ishikawa, Glass transition of associated solvents studied by fluorescence measurement of doped chromophores. Phys. Rev. B 53, 8349-8353 (1996).

8. J. Y. Ye, T. Hattori, H. Nakatsuka, Y. Maruyama, M. Ishikawa, Microscopic dynamics of the glass transition investigated by time-resolved fluorescence measurements of doped chromophores. Phys. Rev. B 56, 5286-5295 (1997).

9. H. Nishimori, G. Ortiz, "2.11 Dynamic critical phenomena" in "Elements of phase transitions and critical phenomena", (Oxford, 2011), pp 46-51.

10. K. Chibisov, G. V. Zakharova, H. Görner, Yu. A. Sogulyaev, I. L. Mushkalo, A. I. Tolmachev, Photorelaxation processes in covalently linked indocarbocyanine and thiacarbocyanine dyes.

J. Phys. Chem. 99, 886-893 (1995).

11. T. Oba, M. Vach, Relaxation in thin films mapped across the film thickness by astigmatic single-molecule imaging. ACS Macro Lett. 1, 784-788 (2012).

12. McKenna, G. B.; Simon, S. L. $50^{\text {th }}$ Anniversary perspective: challenges in the dynamics and kinetics of glass-forming polymers. Macromolecules 50, 6333-6361 (2017).

13. M. Beiner, J. Korus, H. Lockwenz, K. Schröter, E. Donth, Heat capacity spectroscopy compared to other linear response methods at dynamic glass transition in poly(vinyl acetate). Macromolecules 29, 5183-5189 (1996).

14. H. Sillescu, Heterogeneity at the glass transition: a review. J. Non-Cryst. Solids 243, 81-108 (1999).

15. M. D. Ediger, Spatially heterogeneous dynamics in supercooled liquids. Annu. Rev. Phys. Chem. 51, 99-128 (2000).

16. U. Tracht, M. Wilhelm, A. Heuer, H. Feng, K. Schmidt-Rohr, H. W. Spiess, Length scale of dynamic heterogeneities at the glass transition determined by multidimensional nuclear magnetic resonance. Phys. Rev. Lett. 81, 2727-2730 (1998).

17. E. Hempel, G. Hempel, A. Hensel, C. Schick, E. Donth, Characteristic length of dynamic glass transition near $T_{\mathrm{g}}$ for a wide assortment of glass-forming substances. J. Phys. Chem. B 104, 2460-2466 (2000).

18. H. Mori, Transport, collective motion, and Brownian motion. Prog. Theor. Phys. 33, 423455 (1965).

19. H. Shimizu, "Kanwa-gensyo no kiso (Fundamentals in relaxation phenomena)" in Kanwagensyo no kagaku (Relaxation phenomena in chemistry), Eds. K. Higashi, S. Nagakura (Iwanami, 1973), pp 5-73 (in Japanese).

20. H. B. Callen, "Critical phenomena" in "Thermodynamics and introduction to thermostatistics" $2^{\text {nd }}$ Ed. (Wiley, 1985), pp 255-275. 
21. W. Kauzmann, The nature of the glassy state and the behavior of liquids at low temperatures. Chem. Rev. 43, 219-256 (1948).

22. T. R. Kirkpatrick, D. Thirumalai, "Random first-order phase transition theory of the structural glass transition" in "Structural glasses and supercooled liquids", Eds. P. G. Wolynes, V. Lubchenko (Wiley, 2012), pp 223-236.

23. F. Köhn, J. Hofkens, R. Gronheid, M. Van der Auweraer, F. C. De Schryver, Parameters influencing the on- and off-times in the fluorescence intensity traces of single cyanine dye molecules. J. Phys. Chem. A 106, 4808-4814 (2002).

24. H. Mortazavian, C. J. Fennell, F. D. Blum, Structure of the interfacial region in adsorbed poly(vinyl acetate) on silica. Macromolecules 49, 298-307 (2016).

25. K. Jia, Y. Wan, A. Xia, S. Li, F. Gong, G. Yang, Characterization of photoinduced isomerization and intersystem crossing of the cyanine dye Cy3. J. Phys. Chem. A 111, 15931597 (2007).

26. N. J. Turro,"Photophysical radiationless transitions" in Modern Molecular Photochemistry (Addison-Wesley, ed. 2, 1978), pp 153-198.

27. S. Yu. Grebenkin, B. V. Bol'shakov, Cage effects upon light irradiation on azo compounds: cis $\rightarrow$ trans isomerization in polymethyl methacrylate. J. Photochem. Photobiol., A 122, 205-209 (1999). 